\title{
A rapidly enlarging left medial orbital lesion
}

A 49-year-old man presented with a 2-month history of a rapidly enlarging left medial orbital mass. There was no local inflammation, pain, discharge, visual or nasal symptoms. On examination, there was a firm mass bulging between the left nasal bone medially and the left eye measuring $2 \times 1 \mathrm{~cm}$ in dimension (Fig. 1), with no overlying skin paresthesia or cervical lymphadenopathy. No punctum was seen. Nasoendoscopy was normal. There was no ophthalmoplegia or exophthalmos on clinical examination.

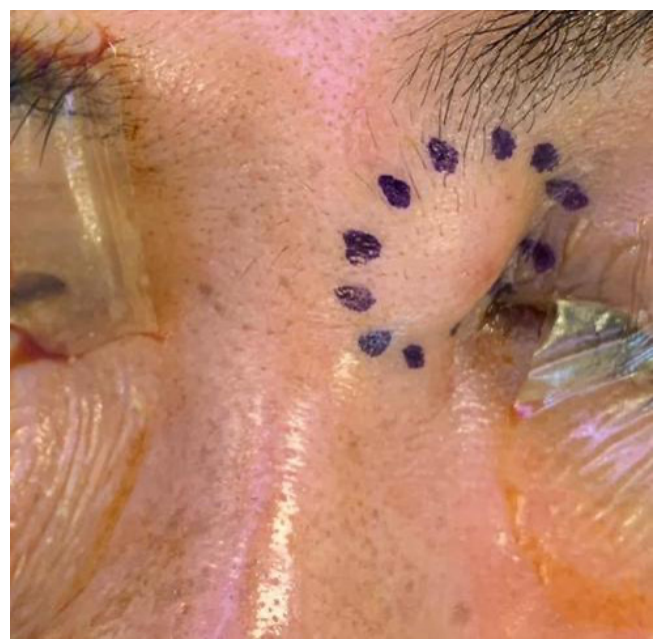

Fig. 1. Clinical photo of the left medial canthal lesion measuring $1.5 \times 1.2 \mathrm{~cm}$. (Colour figure available online.)
Computed tomography (CT) of the face showed a rim-enhancing, hypodense ovoid lesion at the medial aspect of the left orbital rim. Magnetic resonance imaging (MRI) scan showed a mass measuring $1.2 \times 1.1 \times 1.4 \mathrm{~cm}$. It demonstrated intermediate $\mathrm{T} 1-$ weighted and intermediate-to-high T2-weighted signal, with prominent peripheral enhancement (Fig. 2). The mass appeared separate from the left nasolacrimal duct sac. The nasal bone left globe and extraocular muscles were intact.

Fine needle aspiration cytology (FNAC) showed loosely cohesive polygonal spindle cells. Nuclei were bland, often elongated and wavy/buckled in appearance. No high-grade nuclear features were seen.

What is your diagnosis?
A. Lipoma
B. Nodular fasciitis
C. Solitary fibrous tumour
D. Inflammatory pseudotumour (non-specific orbital inflammation)
E. Benign peripheral nerve sheath tumour

Findings and diagnosis. The patient underwent surgical excision via a lynch incision, and a fibrous tumour measuring $1.5 \times 1.2 \mathrm{~cm}$ was resected. It was attached to the medial canthal tendon but not invading the underlying bone. The lacrimal sac was not involved.

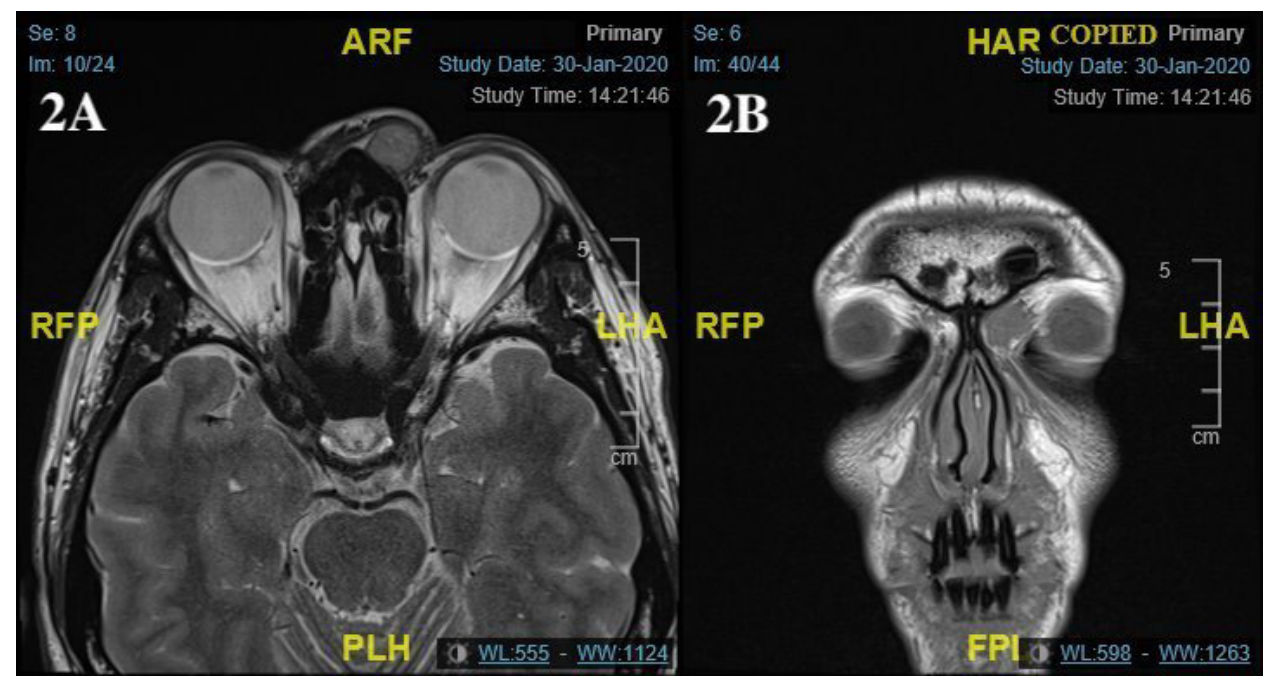

Fig. 2. (A) Axial T2-weighted magnetic resonance imaging (MRI) scan showing intermediate-to-high T2-weighted signal not involving the nasal cavity or the orbit, lying separate from the nasolacrimal duct. (B) Coronal T1-weighted MRI scan with contrast, showing an ovoid superficial lesion at the left nasal bridge with prominent peripheral enhancement, and an intact nasal bone. (Colour figure available online.) 
Histopathological examination showed fascicles of spindle cells in a fibrous stroma exhibiting focal myxoid change with no significant cytologic atypia. Mitoses were noted but no atypical mitoses were seen. Extravasated red blood cells were seen in the stroma. Immunohistochemical studies showed reactivity for smooth muscle actin and negative staining for desmin, CD34, HMB45, S100 protein and pancytokeratin AE1/AE3, in keeping with nodular fasciitis (Fig. 3). Although not done in our patient, when dedicated FNAC sampling with adequate cell block tissue is available, it is possible to obtain a definite diagnosis with molecular testing for USP6 gene rearrangements in an appropriate cytomorphological context.

Discussion. Nodular fasciitis is a benign, self-limiting fibroproliferative disease with nodules most commonly developing in the subcutaneous fascia in the extremities, trunk, or head and neck. It is equally common in men and women, with peak incidence in the third and fourth decades. Antecedent trauma as an inciting factor has been reported in a minority of cases, although evidence for this association is weak. Nodular fasciitis can be painless or tender, and typically presents with a preoperative duration of $<3$ months. ${ }^{1}$ Although benign, its rapid growth can be misdiagnosed as an orbital malignancy, resulting in aggressive treatment that can be disastrous especially in aesthetically prominent areas, such as the periorbital area in this case. ${ }^{1}$

Imaging features of nodular fasciitis are non-specific, thus histological examination is often necessary. ${ }^{2}$ On CT and MRI images, nodular fasciitis is generally seen as a well-defined, superficial soft tissue mass or with variably infiltrative features. As in our patient, moderate to strong enhancement has been commonly reported, although a wide range of degrees of enhancement have been noted. ${ }^{3}$
FNAC can be used as a minimally invasive outpatient procedure, although the diagnosis of nodular fasciitis has multiple pitfalls, as there is no specific cytomorphologic criteria for definitive diagnosis. The cytomorphologic features commonly include plump, spindle-to-stellate cells with bland ovoid nuclei arranged in loose fascicles, and the stroma of the tumour predominantly myxoid or collagenous. Cells notably lack nuclear atypia or pleomorphism but often display a high mitotic rate. ${ }^{1}$ Immunohistochemical studies of nodular fasciitis confirm the myofibroblastic nature of tumour cells that are positive for smooth muscle actin and vimentin; and negative for desmin, cytokeratin AE1/AE3, CD34, S100 protein and HMB45. ${ }^{4}$ Unfortunately, frozen section biopsies of nodular fasciitis show varying results ranging from benign spindle cell neoplasms to an indeterminate diagnosis as morphology overlaps with sarcomas, especially low-grade sarcomas. Hence, the procedure might be of limited value in this context. ${ }^{5}$

Given the clinical features of an orbital smooth firm lump with no overlying skin changes and presenting as a circumscribed ovoid lesion on imaging, our differentials included common benign mesenchymal lesions such as lipomas, benign peripheral nerve sheath tumour, nodular fasciitis, solitary fibrous tumour and inflammatory pseudotumour. Lipomas were ruled out with the lack of both adipocytes on FNAC cytology and characteristic high signals on MRI. Inflammatory pseudotumours featuring bland spindle cells and inflammatory cells are diagnoses of exclusion that have no distinctive radiological or histopathological characteristics. Solitary fibrous tumours are rare spindle cell neoplasms that can arise from nearly anywhere in the body but were unlikely in this case due to the lack of distinct imaging and cytological findings. These include variable mixed intensities on T2-weight MRI, typical cytological

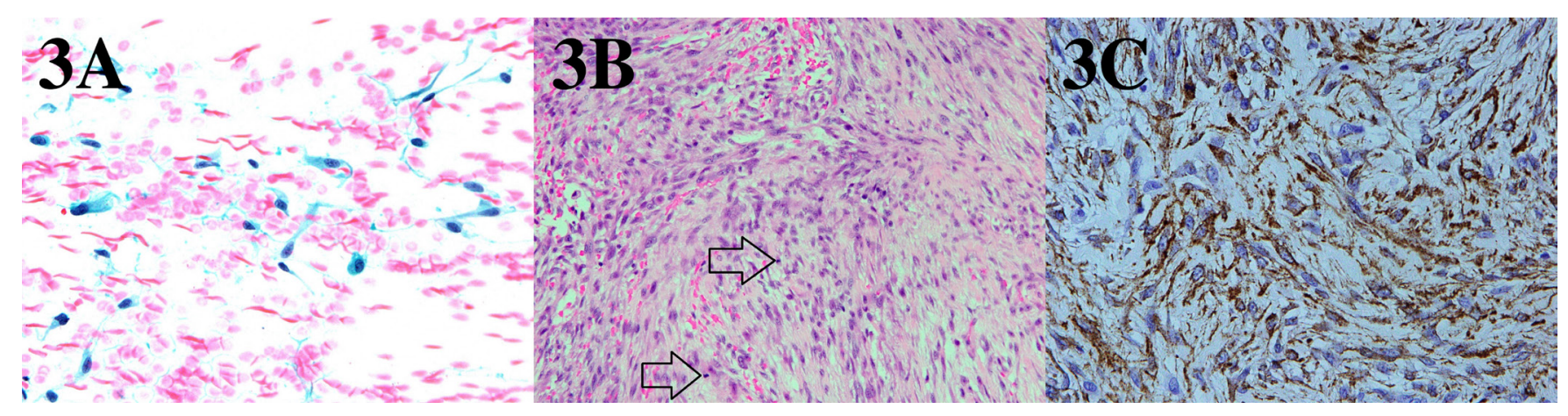

Fig. 3. (A) Loosely dispersed bland plump spindle cells on Papanicolaou stain, 400x magnification. (B) Intersecting fascicles of bland plump spindle cells with extravasated red blood cells and scattered mitoses (arrows), 200x magnification. (C) Spindle tumour cells with positive cytoplasmic immunostaining for smooth muscle actin, 400x magnification. (Colour figure available online.) 
findings such as stripped nuclei, or thick ropy bands of matrix material. Ultimately, FNAC is typically the first-line diagnostic technique for soft tissue swellings, and in our case, showed no high-grade features to narrow down the differential diagnoses to a reactive, benign or low-grade malignant spindle cell lesion. Features present on FNAC raised the possible differentials of benign peripheral nerve sheath tumour, as well as myofibroblastic lesions such as nodular fasciitis. However, histological features with negative S100 immunostaining excluded benign peripheral nerve sheath tumour.

While a specific FNAC-based diagnosis of nodular fasciitis is challenging, recent advancements have shown that cell block ancillary testing for USP6 gene rearrangement may be considered for confirmation of nodular fasciitis. ${ }^{6}$ These were first identified in aneurysmal bone cysts, and recently in nodular fasciitis, myositis ossificans and cellular fibrous tumours of tendon sheath. It suggests a benign and self-limiting condition, hence the final specific diagnosis has to be interpreted in appropriate clinical and cytomorphological contexts. To note, other conditions associated with USP6 gene rearrangements are very rarely described in the head and neck region.

Local excision of the nodular fasciitis is often performed as the disease can be completely resected, after which local recurrence is rare. Conservative management with observation is a possibility, and some cases have been known to resolve spontaneously. ${ }^{7}$ Some studies suggest the use of intralesional steroid injections or laser therapy, which may be useful as alternative treatments in areas where complete excision is difficult, or in aesthetically prominent areas such as the face. ${ }^{8}$ Recurrent masses are so uncommon that malignancies should be considered with careful reassessment of the original diagnosis. ${ }^{9}$ Our patient has been followed up for 1 year with no recurrence of the lesion. He has no epiphora and the incision has healed well.
Nodular fasciitis is an uncommon but possible differential for a subcutaneous periorbital lesion. The correct diagnosis helps in avoiding overtreatment of these lesions.

\section{REFERENCES}

1. Baranov E, Hornick JL. Soft Tissue Special Issue: Fibroblastic and Myofibroblastic Neoplasms of the Head and Neck. Head Neck Pathol 2020;14:43-58.

2. Kim ST, Kim HJ, Park SW, et al. Nodular fasciitis in the head and neck: CT and MR imaging findings. AJNR Am J Neuroradiol 2005;26:2617-23.

3. Shin JH, Lee HK, Cho KJ, et al. Nodular fasciitis of the head and neck: Radiographic findings. Clin Imaging 2003;27:31-7.

4. Nishi SP, Brey NV, Sanchez RL. Dermal nodular fasciitis: three case reports of the head and neck and literature review. J Cutan Pathol 2006;33:378-82.

5. Reitzen SD, Dogan S, Har-El G. Nodular fasciitis: a case series. J Laryngol Otol 2009;123:541-4.

6. Patel NR, Chrisinger JSA, Demicco EG, et al. USP6 activation in nodular fasciitis by promoter-swapping gene fusions. Mod Pathol 2017;30:1577-88.

7. Gelfand JM, Mirza N, Kantor J, et al. Nodular fasciitis. Arch Dermatol 2001;137:719-21.

8. Oh BH, Kim J, Zheng Z, et al. Treatment of Nodular Fasciitis Occurring on the Face. Ann Dermatol 2015;27:694-701.

9. Lee YJ, Kim SM, Lee JH, et al. Nodular Fasciitis of the Periorbital Area. Arch Craniofac Surg 2014;15:43-6.

Rui Tzen Justin Chee ${ }^{1}{ }^{M B B S}$,

Yijin Jereme Gan ${ }^{1}$ MBBS (Hons) (Monash),

Manish Mahadeorao Bundele ${ }^{2}$ FRCPath,

Siu Cheng $\underline{\text { Loke }}^{3}{ }_{F R C R}$, Yuk Ping Cora $\underline{\text { Chau }}{ }^{2}$ FRCPA,

Ming Yann Lim ${ }^{1}$ MBBS (Melbourne)

${ }^{1}$ Department of Otorhinolaryngology, Tan Tock Seng Hospital, Singapore

${ }^{2}$ Department of Pathology, Tan Tock Seng Hospital, Singapore

${ }^{3}$ Department of Diagnostic Radiology, Tan Tock Seng Hospital, Singapore

Correspondence: Dr Ming Yann Lim, Department of Otolaryngology, Head and Neck Surgery, Tan Tock Seng Hospital, 11 Jalan Tan Tock Seng, Singapore 308433 .

Email: ming_yann_lim@ttsh.com.sg 